\title{
An Early Prediction of Lung Cancer using CT Scan Images
}

\author{
Aaron Maurer \\ Information Technology, College of Engineering, University of Missouri. \\ aaronm45@outlookmail.com
}

\author{
Article Info \\ Journal of Computing and Natural Science (http://anapub.co.ke/journals/jens/jens.html) \\ Doi: https://doi.org/10.53759/181X/JCNS202101008 \\ Received 20 October 2020; Revised form 15 November 2020; Accepted 29 December 2020. \\ Available online 05 April 2021. \\ (C2021 Published by AnaPub Publications.
}

\begin{abstract}
Lung cancer is a common occurrence type in a population and one amonglethal cancers. Recently, out of several research presented by diverse health agencies; it is obvious that the fatality ratio is rising due todelayeddiagnosis of lung cancer. Hence, an artificial intelligence-based diagnosis is required to find out the onset of lung nodule micro-calcification, which may support the doctors and radiologists to accurately predict it through image processing methods. In this paper, a novel technique is proposed to identify the nodule micro-calcification pattern by using its physical features. The physical features that considered are the reflection coefficients and mass densities of the binned CT image of lung. The physical features measurements reiteratesonce again the existence of malignant nodule. Then, by applying the methods of thresholding and in interpolation of physical features, a three-dimensional (3D) projected image of the region of interest (ROI) is achieved in respect of physical dimensions. Thus, the nodule size is calculated from 3D projection. This concept is used to verify how best in classification with 100 malignant images (the nodule presence) and 10 normal images (the nodule absence). Apart size measurement, the proposed method supports SVM classifier to act for excellentclassification from normal and malignantinput imagesby just using two physical features. The classifier exhibited an accuracy of $98 \%$.
\end{abstract}

Keywords - Lung cancer, CT image, micro-calcification, reflection coefficient, mass density, tissue impedance

\section{INTRODUCTION}

In general, the pulmonary nodules and micro-calcifications are regularly categorizedbased onspherical opaque bodieswith boundaries of parenchymal lacerations, quantifying with diameter of less $3 \mathrm{~cm}$ [1]. Though, not every pulmonary nodule and micro-calcifications are harmful (depicted in fig 1). Several tinylumped opacities may misrepresent the nodules, specifically when on examined in a one-dimensional plane, however they are featured excellent on multiple planeimages like two-dimension uniform opaque lumps, lesions, plate like atelectasis or respiratory tract anomalies. In medical care, Computer Tomography (CT) takes onto behighly responsive and renowned imaging procedure for detecting and diagnosing the nodule with relative merits on spatial resolution, expense, prevalence, and noninvasiveness [2]. CT is the quite efficient technique to detect the pulmonary nodule and micro-calcifications because of its potential to create 3-dimension images of chest cavity, producing images of nodules, micro-calcifications and pathological tumor at high resolution. An image of CT scan through computer processes in order to support diagnosis of pulmonary nodule has been extensively employed in hospitals. The radiologists require to study variouscharacteristics namely size, location, texture and shape etc. of the nodulesand micro-calcificationsfrom CT scan images to accomplishdiagnosing job. Still, this is quite formidable and laborious, because ofseveral constraints, namely misconception, exhaustiveincluding restricted experience professionally.

Presently, the powerful method in order to detect and evaluate the condition of pulmonary nodule and microcalcificationwould be biopsy technique, howeverthis technique is largely invasive methodthatinvolved extraction of tissue sampledirectly from the nodules and micro-calcifications. With justremoving a smallernodule and microcalcificationpartcannot represent complete characteristics of the nodule precisely because of its heterogeneous features. Hence, sophisticated computer-assisteddiagnosisbased systems will be required to support radiologists to coping these problems, by interpreting the data of diagnosisto take some medical actions. The main 
anomaliesassociated to lung are nodules and calcifications from practitioner's viewpoint. Nodules are unsusceptible in comparison with calcifications. There are two types of calcifications, one as macro and other as micro-calcification. Macro-calcifications [3] considered to be huge calcium deposition in the lung tissue, developed due to many factors. But, smaller calcium depositionstermed micro-calcifications, in the lung tissue cause the highly susceptible to develop cancer.

\section{EXTRACTION METHODS OF IMAGE DETAILS}

Apart the presence of several nodulesand micro-calcifications, particular nodule and micro-calcificationfeatures that determineability of detectioncontain their sizes, shapes, densities, marginalization, sites and association to vessel or pleural systems [4]. Connection with the surfaces of vascular or pleural structuresheightens the chances of nondetection and misapprehensionofpleural swelling or damages. Tiny inadequatelydeterminedlow density nodules and micro-calcification in main sites are increasingly complicated to detect on comparing with superficial bigger nodules and micro-calcificationswell marginalizedscars. The sensitivity of detection of perihilar nodules and microcalcifications was reduced to half in comparison with detection of peripheral nodules [5]. This perihilar nodule and micro-calcification imperceptibility enhanced the sensitivity in little value.

The recognition and classification of small blobs of lung nodules and micro-calcifications from radiological images of thoracic cavityare frequent challenges [6]. Thedecrease in fatality due to lung cancer that proved for wideracceptance of examination of lung cancer has stressed the need foridentification and assessment of nodule [7]. Precise detection ofsubstantial nodulesand micro-calcifications is a precondition that are enhancedon training which used as a technique for computer-assisted detection. Subsequently, the features of detected nodules and microcalcifications exhibits an intrinsic difference. Nodules and micro-calcifications that detected are assessedthrough evaluation by radiologyprimarilyfounded on their shapes and sizes.

\section{Pattern Recognition}

Several image processing methods are beneficial to detect micro-calcification and classify the cancer into benign and malignancy with the features of texture and shape. An extensivepublication reviewdemonstrates that traditional method in detection and classification of micro-calcification includes enhancement [8], accompanied by segmentation [9], next extraction of features [10], and finally the classification [11]. The images of CT [12] and magnetic resonance imaging (MRI) are employed in detection of lung cancer. Initially, the X-ray images are regarded as a mainexamining method to identify any lung anomalies, specifically lung cancer. X-ray is also a type of non-intrusivemethod with the benefits of being less expense, time saving, and greater accuracy. The growth of pattern recognition in image processing requires to be presented in a convenientstyle to support the practitionerfor instantlysuggesting the crucial needed counsel to the patients concerningarea of concern. This paper suggests a unitingthe pattern recognition associated to micro-calcification and modern image processing method to provide a convenientprocedure to a medical practitioner. The suggested technique applies a novel method to estimate the micro-calcification area size by calculating the physical features of the recognizedmicro-calcification in images. In order toextract the lesion physical features in the images, firstly, the binning of inputimageswill beperformed. The method of binning splitsan image into several sub-images, and this is specifically beneficial to reach near to the damaged region and moreovercarry out assessment just on the chosen sub image. The idea of binning is greatly beneficial to study marine images, and here in this paper, this idea is fetched and applied to investigate medical images to assess micro-calcification area size. Further, the reflection coefficient and mass density computations fromlung CT or MRI imagesuse image processing method which is completely uncommon techniqueand adopted as a research tool here.

It is particularly considered here thelung CT imagesto identify the presence of micro-calcification. A tiny calcium deposition that spread in some mannerwhichbecome cancer is termed as micro-calcification. Such calcification is divided into benign and malignant based on its shape, size, boundaries, and spread, and the existence of calcium compound. As an example, the benign cancer is typically due to calcium oxalate, whereas malignancy is due to calcium phosphate. From literature survey, it is found that micro-calcification is one of the main factor that maylead to some cancerous issues. Its identification in beginning and convenient depiction to medicos shall be greatlybeneficial for examining its shape, size, and distribution of the calcium oxalate or calcium phosphate). The micro-calcification seems to beluminous white specks. The intensity of micro-calcification is outweighing the intensity of other zones in the lung image. It is necessary to detect the micro-calcification as region of interest (ROI). This is attained by applying image processing methods to identify the laceration from other tissues. Recognizing the pattern of the laceration is essential for deeper investigation and classification of anomaliesthat occur in lungs. 
The method of recognizing pattern is more helpful in classification of the image features on the basis of commonalities present in some appearances [13]. For identifying the pattern associated to laceration, its related features are to be obtained. Usually, several segmentation methods are applied to detect the ROI.

\section{BACKGROUND AND APPROACH}

The suggested technique is helpful to identify the micro-calcification pattern in the image, which varies from existing traditional methods of pattern recognition. Here, the micro-calcification pattern is determined from its physical features. The physical features that considered are the mass densities and reflection coefficients of nodule region in $\mathrm{CT}$ or MRI images. The identified pattern of micro-calcification is represented in $3 \mathrm{D}$ image form to determine the shape and the size of micro-calcification. The suggested technique comprises 9 stages and they are discussed elaboratelybelow.

\section{Method to measure reflection coefficient}

In lung tissues, a nodulecancerous growth(micro-calcification) is developed due to calcium depositions. If infected lung with such anomaly put underscan, the lacerated bodies reflects more energy. Accordingly, it is possible to calculate the reflection coefficient for the whole image including the lesion part. In the lesion part, the reflected energy and reflection coefficient are both high. An image could be modeled as a two-dimensional function $\mathbf{f}(\mathrm{x}, \mathrm{y})$ with two components as

$$
\mathbf{f}(\mathrm{x}, \mathrm{y})=\mathbf{r}(\mathrm{x}, \mathrm{y}) \mathbf{i}(\mathrm{x}, \mathrm{y})
$$

Where $\mathbf{i}(\mathrm{x}, \mathrm{y})$ is the illumination component and $\mathbf{r}(\mathrm{x}, \mathrm{y})$ is the reflectance component.

$0<\mathrm{i}(\mathrm{x}, \mathrm{y})<\infty, 0<\mathbf{r}(\mathrm{x}, \mathrm{y})<1, \mathrm{r}=0$ represents total absorption, and $\mathbf{r}=1$ represents total reflectance.

\section{Estimating reflection coefficient using curve fitting of least square method}

Once choosing the suitable bin that contains nodule from second level of binning, in order to detect ROI, it is compulsory to evaluate the reflection coefficientsfor setting the threshold. The reflection coefficients for every row is graphed then by regarding the respectivecolumn valuesof bin on $\mathrm{x}$ axis and the spread of the reflection coefficientson y axis.

$$
\sum_{i}\left(\frac{y-y_{i}}{\sigma_{i}}\right)^{2}
$$

Where $y$ is best fitted value, $y_{i}$ is measured data and $\sigma_{i}$ is the standard deviation estimate of $y_{i}$

\section{Mass density measurement}

The mass density is fundamentally a relation by mass per volume. Since the nodule region(micro-calcification) is a calcium deposition, the mass density of it can be determined. Micro-calcification arises due to the calcium deposition namely calcium phosphate and calcium oxalate. The mass of ROI is determinedfrom relevantimages of second-level bin by measuring its respective volume. With the measured values, ROI mass density is evaluated. The evaluated mass density value is depicted.

Reflection coefficient and mass density mapping

The mass densities of complete 12 binned imagesof secondlevel bins are determined and described and the malignant micro-calcifiedROI mass density range lies between 2.7 and $3.1 \mathrm{~g} / \mathrm{cm} 3$. The reflection coefficient range that takes the value from 0.9 to 1 is mapped to the mass density that takes the values from 2.7 to 3.1 and this performance is depicted in Fig. 1. Sinceboth the reflection coefficients and mass densities are determined on the basis of pixel intensities in ROI, the correlation between them happens on linear scale. 


\section{Determination of micro-calcification size}

Since the 3D projection of the binned image can be performed in terms of the distance, the ROI size is conveniently evaluated. In Fig, the $\mathrm{x}$ and $\mathrm{y}$ axes are depicted as the width and height of the binned image and it constitutes a cluster of the micro-calcification.

The method of Interpolation estimates the data at unknown points fromknown neighbor data points. It is hard to locate exact position of micro-calcification in CT images by visual. The image just provides the information on intensities on the basis of the nature of lung tissue. The obtainedCT image when on gray scale conversion, the intensities likely to differ between 0 and 255. Usingthese data and the values of reflection coefficient and mass density, the size of malignant micro-calcificationis calculated possibly fromthe pattern in 3D projection.

\section{Calculating tissue impedance}

The tissue impedances are calculated from reflection coefficients using equation (3). The Fig 1 and 2 depicts the plot of tissue.

$$
\Gamma=\frac{z_{L}-z_{0}}{z_{L}+z_{0}}
$$

In which $\Gamma$ is reflection coefficient, $Z_{0}$ is air impedance which is $376 \Omega$ and $Z_{L}$ is the tissue impedance.

\section{EXPERIMENT RESULTS AND INFERENCES}

The results that follows are discussed in simplified manner. Fig 1 is an input image. The first level binned images of 12 in number are depicted in Fig 2. The 7th binned image comprises micro-calcified ROI. Hence, this sixth image is considered for secondlevel binning and the results of second level binning is depicted in Fig. 2 and bin [2, 2] comprised the micro-calcification nodule.

Reflection Coefficient for second binned image $(2,2)$ of first binned image 11 Reflection Coefficient for second binned image $(2,2)$ of first binned image 11
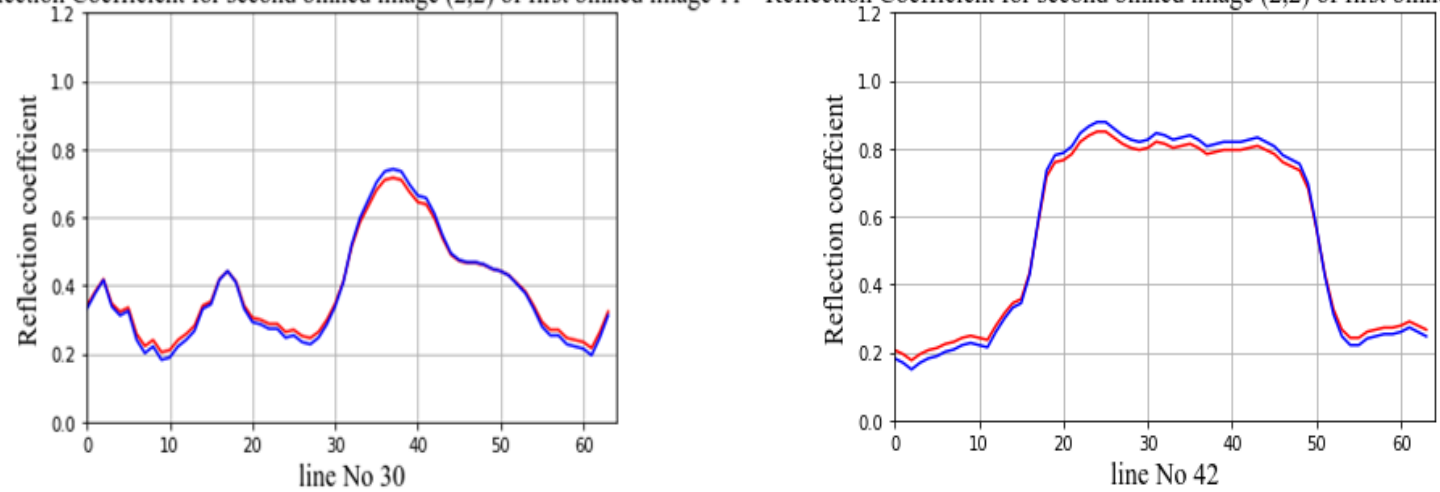

Reflection Coefficient for second binned image $(2,2)$ of first binned image 11 Reflection Coefficient for second binned image $(2,2)$ of first binned image 11
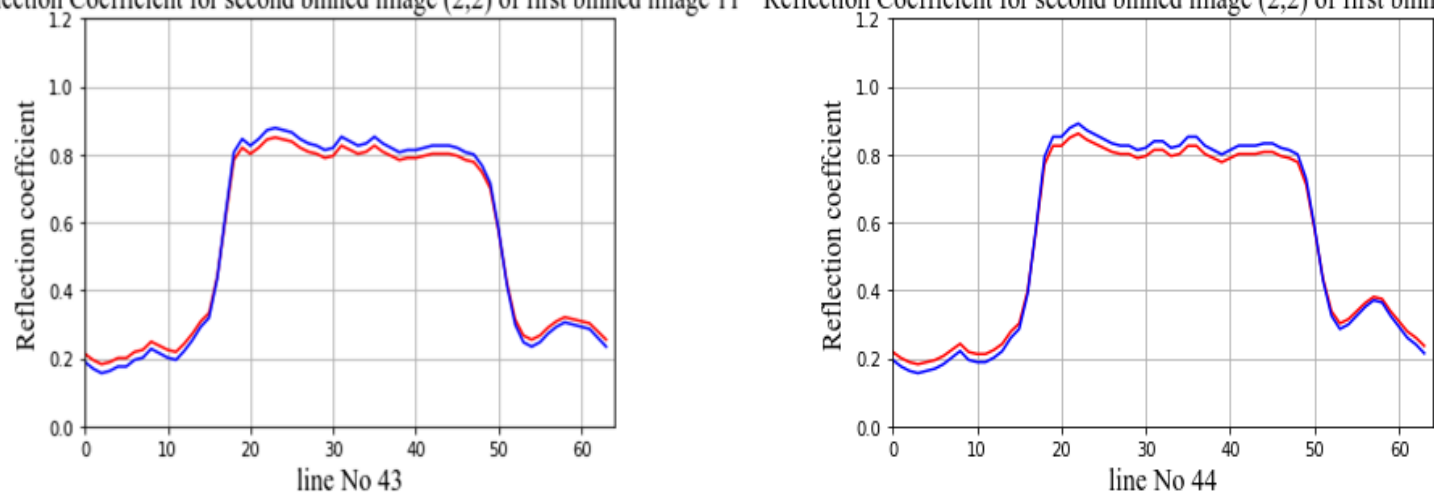

Fig. 1 Reflection coefficient plot for bin [2, 2] with row nos. 30 and 42-44 
Reflection Coefficient for second binned image $(2,2)$ of first binned image 11 Reflection Coefficient for second binned image $(2,2)$ of first binned image 11
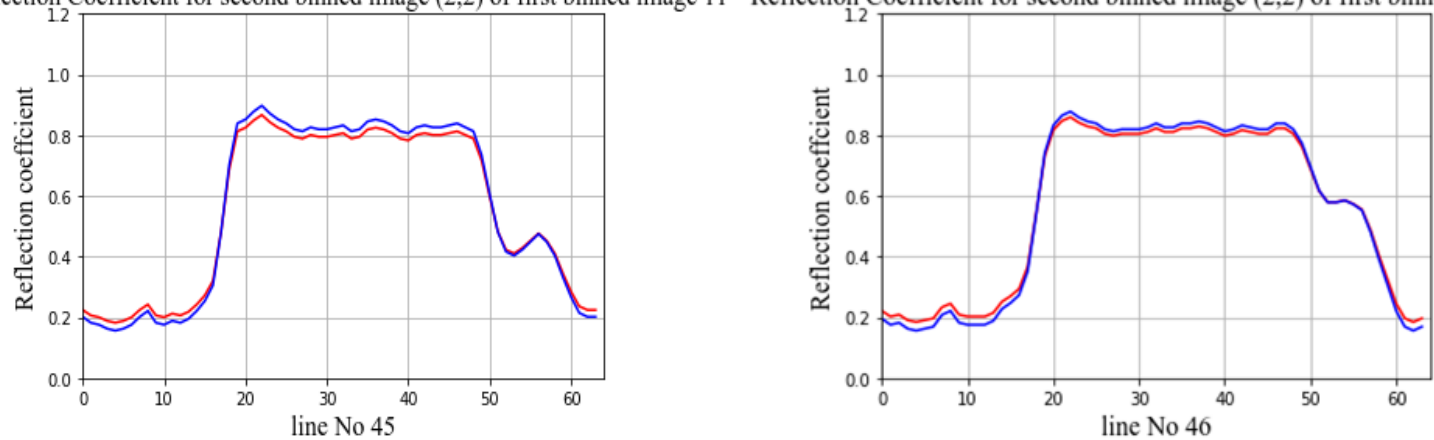

Reflection Coefficient for second binned image $(2,2)$ of first binned image 11 Reflection Coefficient for second binned image $(2,2)$ of first binned image 11
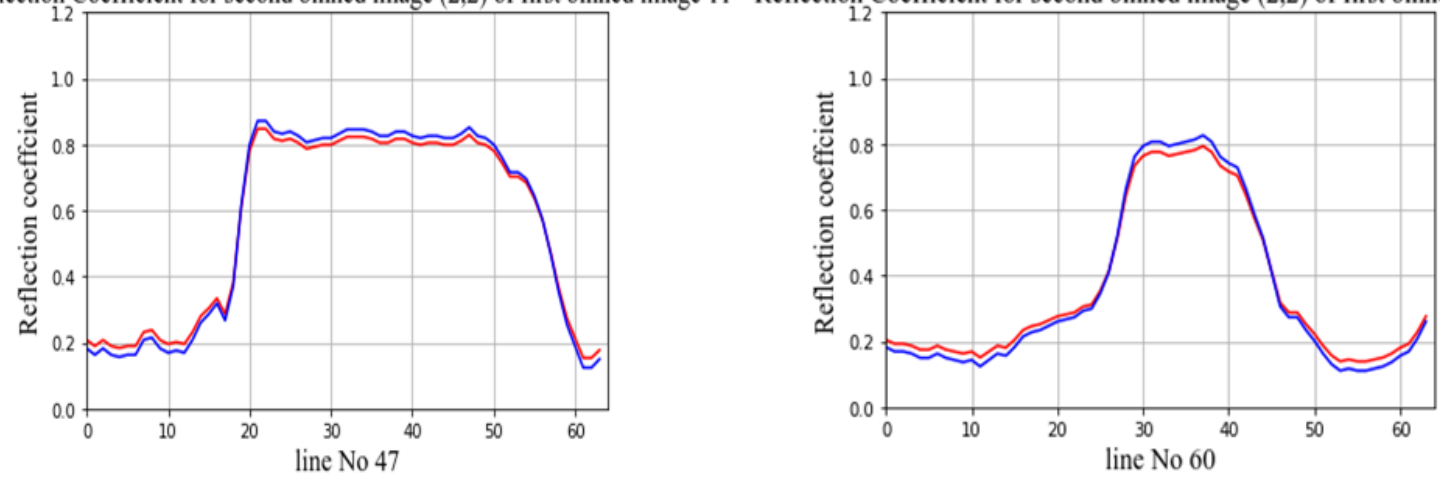

Fig. 2 Reflection coefficient plot for bin $[2,2]$ with row nos. $45-47$ and 60

\section{CONCLUSION}

This investigationsuggested a new automation method with CAD operation namely binning computation of the reflection coefficients and mass densities to derive a $3 \mathrm{D}$ projection of nodule pattern from lung $\mathrm{CT}$ images. In segmenting the nodule successfully, thresholding on the basis of the reflection coefficient was performed. Additionally, using mass density values, the type of calcium deposit in the lung can be determined. The computation of nodule size was carried out using interpolation over image intensity and the reflection coefficient. Therefrom, the nodule was preciselyidentifiedusing $3 \mathrm{D}$ projection of it, and thereby its size was accurately detected. The recognition method applied for investigation aimed majorlyby using physical features namelyreflection coefficient and mass density. Hence this method is unique, and hasbeen unapplied or unstudied comprehensively in any of earlier researches. The prediction between normal and malignancy during classification achieved the accuracy with $98 \%$. Therefore, the suggested method is demonstrated as an efficient techniquefor diagnoses and classification of the lung cancer. The $3 \mathrm{D}$ projection of nodule is a convenient graphical representation, which is so easy to comprehend the nodule size and shape by doctors, radiologists and patients.

\section{References}

[1]. S. Kaur and G. Jindal, "Watershed Segmentation of Lung CT Scan Images for Early Diagnosis of Cancer," International Journal of Computer and Electrical Engineering, pp. 850-852, 2011.

[2]. D. Mhaske, K. Rajeswari, and R. Tekade, "Deep Learning Algorithm for Classification and Prediction of Lung Cancer using CT Scan Images," 2019 5th International Conference On Computing, Communication, Control And Automation (ICCUBEA), Sep. 2019.

[3]. Hoque, A. K. M. A. Farabi, F. Ahmed, and M. Z. Islam, "Automated Detection of Lung Cancer Using CT Scan Images," 2020 IEEE Region 10 Symposium (TENSYMP), 2020.

[4]. D. Sharma and G. Jindal, "Computer Aided Diagnosis System for Detection of Lung Cancer in CT Scan Images," International Journal of Computer and Electrical Engineering, pp. 714-718, 2011.

[5]. M. Muthuraman and S. Ravichandran, "A Novel Automated CNN Based Lung Cancer Prediction Technique (CNNLCPT) for CT scan images," International Journal of Computer Sciences and Engineering, vol. 7, no. 5, pp. 1440-1444, May 2019.

[6]. Nagarajan, "Image processing techniques for analyzing CT scan images towards the early detection of lung cancer," Bioinformation, vol. 15, no. 8, pp. 596-599, Aug. 2019. 
[7]. M. Z. A. Manzoor Ahmed Khawaja, "Effectual lung segmentation for CAD systems using CT scan images," 8th International Multitopic Conference, 2004. Proceedings of INMIC 2004.

[8]. N. A, "Machine Learning Approach to Predict Lung Cancer using CT scan Images," International Journal of Advanced Trends in Computer Science and Engineering, vol. 8, no. 6, pp. 2974-2976, Dec. 2019.

[9]. Q. Firdaus, R. Sigit, T. Harsono, and A. Anwar, "Lung Cancer Detection Based On CT-Scan Images With Detection Features Using Gray Level Co-Occurrence Matrix (GLCM) and Support Vector Machine (SVM) Methods," 2020 International Electronics Symposium (IES), Sep. 2020.

[10]. Kulkarni and A. Panditrao, "Classification of lung cancer stages on CT scan images using image processing," 2014 IEEE International Conference on Advanced Communications, Control and Computing Technologies, May 2014.

[11]. M. Islam, A. H. Mahamud, and R. Rab, "Analysis of CT Scan Images to Predict Lung Cancer Stages Using Image Processing Techniques," 2019 IEEE 10th Annual Information Technology, Electronics and Mobile Communication Conference (IEMCON), Oct. 2019.

[12]. F. Shafiei and S. Fekri-Ershad, "Detection of Lung Cancer Tumor in CT Scan Images Using Novel Combination of Super Pixel and Active Contour Algorithms," Traitement du Signal, vol. 37, no. 6, pp. 1029-1035, Dec. 2020. 